\title{
Stereoregularities of Ring-containing Polymethacrylates Obtained by Radical Polymerization
}

\author{
Jun Nishino, Hisataka Nakahata, and Yasuyoshi Sakaguchi \\ Department of Applied Chemistry, Konan University, Kobe, Japan.
}

(Received September 7, 1970)

\begin{abstract}
Phenyl, 2-naphthyl(2-Np-MA), 1-naphthyl(1-Np-MA), 9-fluorenyl, 5,6,7, 8-tetrahydro-1-naphthyl, cyclohexyl(CH-MA), cyclopentyl(CP-MA), and decahydro-2naphthyl(D-MA) methacrylates were polymerized in bulk, benzene, and $n$-hexane by radical initiators. The polymers were converted into poly(methyl methacrylate)(PMMA) by hydrolysis followed by methylation. The tacticities of the polymers were studied by comparing the IR and NMR spectra of the derived PMMA's. The polymers containing aromatic substituents were more isotactic than conventional PMMA, and especially poly(2-Np-MA) prepared in $n$-hexane and poly(1-Np-MA)'s were considerably isotactic. On the other hand, tacticities of poly(CH-MA)'s, poly(CP-MA)'s, and poly(D-MA)'s were nearly the same as those of conventional PMMA. These results suggest that aromatic rings of the methacrylates seem to promote the formation of isotactic sequence and this effect is enhanced with naphthyl groups, but the aliphatic rings have little effect on such stereoregulation.
\end{abstract}

KEY WORDS Stereoregularity / Radical Polymerization / Poly(phenyl methacrylate)/Poly(2-naphthyl methacrylate)/Poly(1-naphthyl methacrylate) / Poly $(5,6,7,8$-tetrahydro-1-naphthyl methacrylate) / Poly(cyclohexyl methacrylate)/ Poly(cyclopenthyl methacrylate)/ Poly(decahydro-2-naphthyl methacrylate) / Poly(methyl methacrylate) / IR / NMR /

Many authors ${ }^{1}$ have reported that most poly(methacrylic acid)(PMAA) and poly(methyl methacrylate)(PMMA) obtained by radical polymerization of the corresponding monomers are highly syndiotactic. The effect of the polymerization medium on stereoregularities of resulting PMMA's was also investigated by these authors ${ }^{1}$, but the effect was slight.

The stereoregularities of polymers obtained by radical polymerization from methacrylic monomers containing aromatic substituents would be noteworthy from the fact that syndiotacticities of $\operatorname{poly}(\alpha \text {-methylbenzyl methacrylate })^{2,3,4}$ and poly $(\beta \text {-phenylethyl methacrylate })^{3}$ are higher, but those of $\operatorname{poly}(\alpha$-phenylpropyl methacrylate) and poly(2,4,6-triphenylbenzyl methacrylate $)^{3,5}$ are lower than those of conventional PMMA. Recently, when we were carring out this work, Yuki and coworkers ${ }^{6,7}$ reported that benzyl and diphenylmethyl methacrylates behave similarly to methyl methacrylate but trityl methacrylate gives a highly isotactic polymer by radical poly- merization. As mentioned above, the stereoregularity of methacrylic polymer prepared by radical polymerization depends on the structure of substituents in the parent monomer, but the relation between the structure of the monomer and the stereoregularity of the polymer prepared therefrom has not yet been clarified.

To get some information on the factors controlling the stereoregularity of methacrylic polymer during radical polymerization, in the present investigation phenyl(Ph-MA), 2-naphthyl(2-Np-MA), 1-naphthyl(1-Np-MA), 9-fluorenyl(F-MA), 5, 6, 7, 8-tetrahydro-1-naphthyl(T-MA), cyclohexyl(CH-MA), cyclopentyl(CP-MA), and decahydro-2-naphthyl(D-MA) methacrylates were synthesized and polymerized by radical initiators under several conditions. The polymers obtained were converted into PMMA's by hydrolysis followed by methylation with diazomethane. Stereoregularities of the original polymers were investigated with infrared(IR) and nuclear magnetic resonance(NMR) spectra of the derived PMMA's. 


\section{EXPERIMENTAL}

\section{Materials}

Ph-MA, 2-Np-MA, and 1-Np-MA were prepared by the reaction of methacrylyl chloride with the corresponding phenols in 5-\% sodium hydroxide aqueous solution cooled in an ice-bath according to the procedure of Patai. ${ }^{8}$ Ph-MA was redistilled at $64-66^{\circ} \mathrm{C}(2 \mathrm{~mm}) ; n_{\mathrm{D}}^{20} 1.5149$; yield $75 \%$. The values for this monomer prepared by pyrolysis of phenyl- $\alpha$-acetoxy isobutylate are bp $47 \sim 50^{\circ} \mathrm{C}(0.2 \mathrm{~mm})$ and $n_{\mathrm{D}}^{20} 1.5147 .^{9} \quad 2-$ Np-MA was recrystallized from 70- $\%$ ethanol, $\mathrm{mp} 65-66^{\circ} \mathrm{C}$; lit. $^{8} 66^{\circ} \mathrm{C}$. 1-Np-MA was redistilled at $137-140^{\circ} \mathrm{C}(4 \mathrm{~mm})$; yield $71 \%$.

Anal. Calcd for $\mathrm{C}_{14} \mathrm{H}_{12} \mathrm{O}_{2} ; \mathrm{C}, 79.22 ; \mathrm{H}, 5.70$, Found: C, 78.80, H, 5.91.

F-MA and T-MA were obtained by the reaction of methacrylyl chloride with 9-fluorenol or 5 , 6, 7, 8-tetrahydro-1-naphthol in pyridine with stirring at $0^{\circ} \mathrm{C}$ for $2 \mathrm{hr}$ and at room temperature for another $2 \mathrm{hr}$. F-MA was recrystallized from $70-\%$ ethanol, mp $60-62^{\circ} \mathrm{C}$; yield $45 \%$.

Anal. Calcd for $\mathrm{C}_{17} \mathrm{H}_{14} \mathrm{O}_{2}:$ C, 81.57; H, 5.64. Found: C, 80.98; H, 5.80.

T-MA was redistilled at $130-135^{\circ} \mathrm{C}(3 \mathrm{~mm})$; yield $36 \%$.

Anal. Calcd for $\mathrm{C}_{14} \mathrm{H}_{16} \mathrm{O}_{2}:$ C, 77.75; H, 7.46. Found: C, 77.53; H, 7.80. Fluorenol was prepared from fluorene by oxidation. ${ }^{10}$

CH-MA, OP-MA, and D-MA were prepared by refluxing the corresponding cyclic alcohols and methacrylic acid with $p$-toluensulfonic acid in benzene for $10 \mathrm{hr}$. CH-MA: bp $71-74^{\circ} \mathrm{C}$ (5 mm); lit. ${ }^{11} 71-74^{\circ} \mathrm{C}(5 \mathrm{~mm})$. CP-MA: bp 76 $-78^{\circ} \mathrm{C}(20 \mathrm{~mm})$; 1 it. $.^{12} 70-72^{\circ} \mathrm{C}(22 \mathrm{~mm})$. D-MA: bp $127-130^{\circ} \mathrm{C}(6 \mathrm{~mm})$; yield $53 \%$.

Anal. Calcd for $\mathrm{C}_{14} \mathrm{H}_{22} \mathrm{O}_{2}: \mathrm{C}, 75.63 ; \mathrm{H}, 9.98$. Found: C, 75.27; H, 10.02.

Benzene and $n$-hexane were purified in the usual manner ${ }^{13}$ and the other reagents and initiators were of guaranteed grade. Benzene solution of diazomethane was prepared by treating nitrosomethylurea with potassium hydroxide aqueous solution in benzene. ${ }^{14}$

\section{Polymerization}

The required amounts of monomer, initiator, and solvent were put into an ampoule. After flushing with nitrogen, the ampoule was sealed off and placed into a thermostat to carry out polymerization. The resulting polymers prepared in benzene or bulk were isolated by pouring the contents of the ampoules into methanol containing hydroquinone. The polymers obtained in $n$-hexane and in ethanol precipitated during the polymerization. The polymers were further purified by reprecipitation from the benzenemethanol system.

\section{Conversion of the Polymers to PMMA's}

The polymers were hydrolyzed to PMAA's with 10 parts of concentrated sulfuric acid for two days at $50^{\circ} \mathrm{C}$ under a nitrogen atomosphere, and refluxed in 10-\% sodium hydroxide aqueous solution for another two days. The PMAA's obtained were purified by means of dialysis and ion-exchange of their aqueous solutions. The PMAA's were then methylated with diazomethane in benzene. ${ }^{15}$ The derived PMMA's were isolated by pouring the benzene solution into methanol.

\section{Measurements}

The IR spectra were recorded with a Hitachi EPI-S2 Spectrophotometer as $\mathrm{KBr}$ disk. The NMR spectra were recorded with a Hitachi PerkinElmer R-20 Spectrometer at $60 \mathrm{MHz}$ using tetramethylsilane as an internal reference in deuterochloroform at $80^{\circ} \mathrm{C}$. The tacticity of the derived PMMA was determined as described by Bovey and Tiers. ${ }^{16}$

\section{RESULTS}

Polymerization conditions and results are listed in Table I. When the polymerizations were carried out in $n$-hexane and in ethanol, the polymers separated out from the medium in the course of the polymerization processes. The IR spectra of the polymers obtained from the same monomer were practically the same as each other regardless of the polymerization conditions. The IR spectra of the derived PMAA's indicated that the hydrolysis was complete. The typical IR spectra of the derived PMMA's are shown in Figure 1. As shown therein the conversion to PMMA's was complete and these PMMA's were free from any impurity and unusual structure. It has been usually admitted that absorption peaks in an IR spectrum of conventional (fairly 


\section{Stereoregularities of Polymethacrylates}

Table I. Conditions and results of polymerization

\begin{tabular}{|c|c|c|c|c|c|c|c|c|c|c|}
\hline \multirow{2}{*}{ No. } & \multirow{2}{*}{$\begin{array}{c}\text { Monomer, } \\
\mathrm{g}\end{array}$} & \multirow{2}{*}{$\begin{array}{l}\text { Initiator, }{ }^{\mathrm{a}} \\
\mathrm{mg}\end{array}$} & \multirow{2}{*}{$\begin{array}{l}\text { Solvent, } \\
\mathrm{m} l\end{array}$} & \multirow{2}{*}{$\underset{{ }^{\circ} \mathrm{C}}{\text { Temp, }}$} & \multirow{2}{*}{$\begin{array}{c}\text { Time, } \\
\text { day }\end{array}$} & \multirow{2}{*}{$\underset{\%}{\text { Yield, }}$} & \multicolumn{4}{|c|}{ Tacticity, \% } \\
\hline & & & & & & & $I$ & $H$ & $S$ & $4 I S / H^{2}$ \\
\hline 1 & $\mathrm{Ph}-\mathrm{MA}(10)$ & $\mathrm{A}(50)$ & $\mathrm{B}(20)$ & 60 & 7 & 98 & 6 & 35 & 59 & 1.2 \\
\hline 2 & $\mathrm{Ph}-\mathrm{MA}(5)$ & $\mathrm{B}(50)$ & $\mathrm{H}(20)$ & 60 & 2 & 90 & 16 & 39 & 45 & 1.9 \\
\hline 3 & $2-\mathrm{Np}-\mathrm{MA}(5)$ & $\mathrm{B}(50)$ & none & 70 & 10 & 80 & 14 & 39 & 47 & 1.7 \\
\hline 4 & $2-\mathrm{Np}-\mathrm{MA}(10)$ & $\mathrm{A}(50)$ & $\mathrm{B}(20)$ & 60 & 7 & 99 & 12 & 38 & 50 & 1.7 \\
\hline 5 & $2-\mathrm{Np}-\mathrm{MA}(5)$ & $\mathrm{A}(50)$ & $\mathrm{E}(20)$ & 60 & 10 & 85 & 14 & 40 & 46 & 1.6 \\
\hline $6-1$ & $2-\mathrm{Np}-\mathrm{MA}(5)$ & $B(50)$ & $\mathrm{H}(20)$ & 60 & 4 & 60 & 58 & 24 & 18 & 7.2 \\
\hline $6-2$ & $2-\mathrm{Np}-\mathrm{MA}(5)$ & $\mathrm{B}(50)$ & $\mathrm{H}(20)$ & 80 & 0.1 & 20 & 24 & 39 & 37 & 2.3 \\
\hline $6-3^{c}$ & 2-Np-MA( 5$)$ & $\mathrm{B}(50)$ & $\mathrm{H}(20)$ & 80 & 0.2 & 45 & 17 & 37 & 46 & 2.3 \\
\hline 7 & $1-\mathrm{Np}-\mathrm{MA}(12)$ & $\mathrm{A}(120)$ & none & 80 & 0.1 & 67 & 41 & 35 & 24 & 3.2 \\
\hline 8 & $1-\mathrm{Np}-\mathrm{MA}(5)$ & $A(200)$ & $\mathrm{B}(45)$ & 60 & 4 & 80 & 34 & 40 & 26 & 2.2 \\
\hline $9-1$ & $1-\mathrm{Np}-\mathrm{MA}(5)$ & $\mathrm{B}(50)$ & $\mathrm{H}(75)$ & 80 & 2 & 70 & 37 & 38 & 25 & 2.5 \\
\hline $9-2^{c}$ & $1-\mathrm{Np}-\mathrm{MA}(5)$ & $\mathrm{B}(50)$ & $\mathrm{H}(75)$ & 80 & $0 . \overline{1}$ & 28 & 35 & 39 & 26 & 2.4 \\
\hline 10 & F-MA( 4$)$ & $\mathrm{B}(20)$ & none & 80 & 2 & 82 & 13 & 42 & 45 & 1.3 \\
\hline 11 & F-MA( 4$)$ & $\mathrm{B}(100)$ & $\mathrm{B}(56)$ & 80 & 2 & 90 & 10 & 41 & 49 & 1.2 \\
\hline 12 & F-MA( 3$)$ & $\mathrm{B}(100)$ & $\mathrm{H}(57)$ & 80 & 2 & 75 & 10 & 39 & 51 & 1.3 \\
\hline 13 & $\mathrm{~T}-\mathrm{MA}(2)$ & $\mathrm{A}(20)$ & none & 80 & 0.1 & 90 & 12 & 46 & 42 & 1.0 \\
\hline 14 & $\mathrm{~T}-\mathrm{MA}(2)$ & $\mathrm{B}(45)$ & $\mathrm{H}(50)$ & 80 & 2 & 35 & 11 & 44 & 45 & 1.0 \\
\hline 15 & CP-MA( 5 ) & $\mathrm{B}(50)$ & $\mathrm{B}(20)$ & 60 & 3 & 90 & 5 & 35 & 60 & 1.1 \\
\hline 16 & CP-MA( 5$)$ & $\mathrm{B}(50)$ & $\mathrm{H}(20)$ & 60 & 3 & 90 & 6 & 36 & 58 & 1.1 \\
\hline 17 & $\mathrm{CH}-\mathrm{MA}(5)$ & $\mathrm{B}(50)$ & $\mathrm{B}(20)$ & 60 & 3 & 90 & 5 & 34 & 61 & 1.1 \\
\hline 18 & $\mathrm{CH}-\mathrm{MA}(5)$ & $\mathrm{B}(50)$ & $\mathrm{H}(20)$ & 60 & 3 & 85 & 4 & 32 & 64 & 1.0 \\
\hline 19 & D-MA( 5 ) & $\mathrm{A}(20)$ & none & 60 & 0.5 & 90 & 6 & 36 & 58 & 1.1 \\
\hline 20 & D-MA( 9 ) & $\mathrm{A}(200)$ & $\mathrm{B}(71)$ & 60 & 6 & 75 & 6 & 33 & 61 & 1.2 \\
\hline 21 & D-MA $(5)$ & $\mathrm{B}(100)$ & $\mathrm{H}(45)$ & 60 & 7 & 60 & 6 & 38 & 56 & 0.9 \\
\hline
\end{tabular}

a $\mathrm{A}$, azobisisobutyronitrile; $\mathrm{B}$, benzoyl peroxide.

b $\mathrm{B}$, benzene; $\mathrm{E}$, ethanol; $\mathrm{H}, n$-hexane.

c These polymerizations were carried out while being stirred.

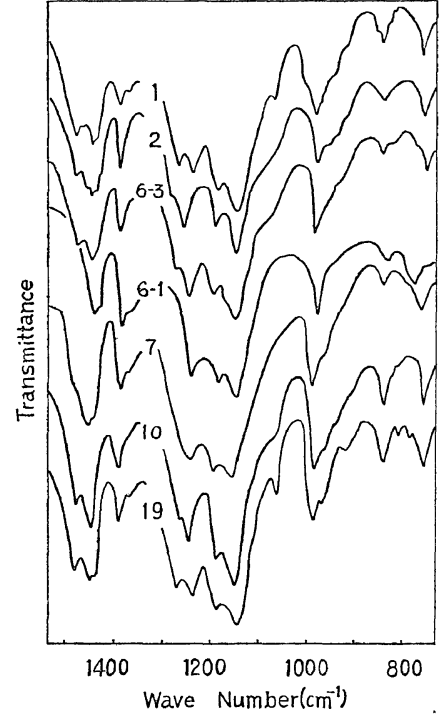

Figure 1. IR spectra of the PMMA's derived from the polymers listed in Table I.



Figure 2. NMR spectra of the PMMA's derived from the polymers listed in Table $I$. 


\section{J. Nishino, H. Nakahata, and Y. Sakaguchi}

syndiotactic) PMMA at 750, 806, 824, 910, 1060, 1280 , and $1475 \mathrm{~cm}^{-1}$ disappear or shift in the case of an isotactic one. ${ }^{17}$ It was found by comparing the above peaks that isotacticities of no. $2-14$ are higher than those of no. 1, 15-21 which are nearly the same as those of conventional PMMA.

The typical NMR spectra of the derived PMMA's are shown in Figure 2. The tacticities in triad determined from the NMR spectra are also given in Table I. As suggested in the IR spectra of the PMMA's, the tacticities of no. 1, 15-21 are similar to those of conventional PMMA, and no. $2-14$, especially no. 6-1, 6-2, 7-9, are more isotactic.

\section{DISCUSSION}

On the basis of these results, it is revealed that the polymers no. 2-14 are more isotactic than conventional PMMA. Aromatic substituents in the monomers, generally prevent the formation of syndiotactic sequences and encourage isotactic ones in radical polymerization and this effect is enhanced with the naphthyl group. Bulkiness or steric hindrance of the substituents would be considered for this effect but CP-MA, CH-MA, and D-MA behave similarly to methyl methacrylate in spite of their bulky ring substituents. A comparison of 2-Np-MA and D-MA, or 1-Np-MA and T-MA, also shows that the effect of bulkiness of alicyclic substituents is very small.

As is well known, tacticity of polymers is greatly affected by the medium in ionic polymerization. Szwarc $^{18}$ had suggested that polymerization in a poor solvent may encourage regular growth because of the necessity of conforming to a helical chain growth pattern. Our present data on no. 2 and 6 show that, even in radical polymerization, tacticity of the polymer prepared in a bad solvent may be considerably different from that of one prepared in a good solvent. Based on the results of no. 6 , when the polymer separates out from the medium, tacticity of the polymer seems to be affected by small variations in the state of the precipitate and consequently the polymerization conditions. Tacticity of the polymers obtained by precipitation polymerization should be further examined.
The values in the last column of Table I can be taken as an indication of the mechanisms of stereoregulation. The values of the polymers with high isotacticity tend to deviate from unity. Therefore the stereoregulation in the polymerization in which relatively isotactic polymers were obtained, cannot be described by a single parameter process. ${ }^{15}$

Although it is not easy at present to determine the exact role of the substituents of the methacrylates on stereoregulation in radical polymerization, the high isotacticities of no. 6-1, 6-2, 7, 8, 9-1, and 9-2 seem to be caused mainly by the aromaticity of the naphthyl substituents. In radical polymerization of methacrylic monomer, the aromatic ring of the monomers seems to promote the formation of isotactic sequence through interaction among them. In order to establish the effect of the cyclic substituent of monomer on stereoregularity of the polymer obtained by radical polymerization, other ringcontaining methacrylic, acrylic, and vinyl monomers should be examined.

Acknowledgment. The authors are much indebted to Mr. M. Matsuoka of the University of Osaka Prefecture for the measurements of NMR spectra.

\section{REFERENCES}

1. H. Watanabe, Kogyo Kagaku Zasshi (J. Chem. Soc. Japan, Ind. Chem. Sect.), 64, 1851 (1961); H. Watanabe, Y. Kato, and A. Nishioka, ibid., 65, 273 (1962); T. G Fox and H. W. Scheneko, Polymer, 3, 575 (1962); I. Sakurada, Y. Sakaguchi, and G. Ohara, Kobunshi Kagaku (Chem. High Polymers, Japan), 19, 704 (1962); I. Sakurada, Y. Sakaguchi, J. Nishino, and T. Iwagaki, ibid., 20, 456 (1963); G. Schroder, Makromol. Chem., 97, 232 (1966); A. Yamada, M. Yanagita, S. Arano, and H. Sekimura, Kogyo Kagaku Zasshi (J. Chem. Soc. Japan, Ind. Chem. Sect.), 73, 2352 (1970).

2. K. Matsuzaki, A. Ishida, N. Tateno, T. Asakura, A. Hasegawa, and M. Tameda, Kogyo Kagaku Zasshi (J. Chem. Soc. Japan, Ind. Chem. Sect.), 68, 852 (1965).

3. K. Yokota and Y. Ishii, J. Polym. Sci., Part B, 3, 771 (1965); Kogyo Kagaku Zasshi (J. Chem. Soc. Japan, Ind. Chem. Sect.), 69, 1053 (1966). 4. K. J. Liu, J. S. Lignowski, and R. Ulman, 
Makromol. Chem., 105, 8 (1967).

5. H. Imai, T. Makimoto, and T. Tsuruta, Kogyo Kagaku Zasshi (J. Chem. Soc. Japan, Ind. Chem. Sect.), 68, 1947 (1965).

6. H. Yuki, K. Hatada, T. Ninomi, and $Y$. Kikuchi, Polymer J., 1, 36 (1970).

7. H. Yuki, K. Hatada, Y. Kikuchi, and T. Ninomi, J. Polym. Sci., Part B, 6, 753 (1968).

8. S. Patai, M. Bentor, and M. E. Reichmann, J. Amer. Chem. Soc., 74, 845 (1952).

9. E. M. Filachione, J. H. Lengel, and W. P. Ratchford, ibid., 72, 840 (1950).

10. “Yuki Kagobutsu Gosei-ho", Vol. 11, Yuki Gosei Kagaku Kyokai Ed., Giho-do, Tokyo, 1968, pp 18 and 93.

11. C. E. Rehberg and W. A. Faucette, J. Amer. Chem. Soc., 72, 4307 (1950).

12. J. Lai and R. Green, J. Org. Chem., 20, 397
(1955).

13. A. Weissberger and E. S. Proskauer, "Organic Solvents"' 2nd ed, Interscience Publishers Inc., New York N.Y., 1955.

14. "Organic Syntheses," Collective, Vol. 2, J. Wily Publishers, New York, N.Y., 1943, pp 165 and 461.

15. A. Katchalsky and H. Eisenberg, J. Polym. Sci., 6, 145 (1951).

16. F. A. Bovey and G. V. D. Tiers, ibid., 44, 173 (1960).

17. U. Baumann, H. Schreiber, and K. Tessmar, Makromol. Chem., 36, 81 (1960); J. Furukawa, T. Tsuruta, S. Nakayama, A. Kawasaki, and G. Wasai, Kogyo Kagaku Zasshi (J. Chem. Soc., Japan, Chem. Ind. Sect.), 64, 591 (1961).

18. M. Szwarc, Chem. Ind. (London), 1958, 1589. 